\title{
Analysis of Vehicle Modification Practice in Bangladesh
}

\author{
Sakib Mahmud Khan ${ }^{1}$ and Md. Shamsul Hoque ${ }^{2}$ \\ 1. Glenn Department of Civil Engineering, Clemson University, Clemson 29634, USA \\ 2. Department of Civil Engineering, Bangladesh University of Engineering and Technology, Dhaka 1000, Bangladesh
}

\begin{abstract}
With the shocking present vehicle growth over 10\%, many vehicles are found to operate within urban areas and national highways of Bangladesh without having necessary registration and fitness papers. Uncontrolled vehicle modification practice has become a serious concern particularly for large vehicles, which leads the vehicle owners not to properly adhere to the vehicle fitness rules. The primary objective of this research is to reveal the extent of vehicle modification of different categories of vehicle. In this regard, field survey is conducted in major bus terminals and truck depots of Dhaka city to find out the issues related to rampant vehicle modification. Later statistical analysis is performed to obtain the most observed vehicle dimensions followed by local body workers. Finally, detailed questionnaire survey is performed on vehicle owners as well as the body makers as an attempt to reveal the poor quality of work performed by unskilled local workers. This paper would describe the findings of this research work along with appropriate remedial measures to control the unsafe modification of vehicles.
\end{abstract}

Key words: Vehicle modification, vehicle fitness, in-vehicle unsafe material, body maker of Bangladesh.

\section{Introduction}

Presence of defective and road unworthy vehicles plying on road poses serious threat to the safety issue of road system in Bangladesh. According to the statistics of BRTA (Bangladesh Road Transport Authority), total number of registered vehicles in Bangladesh up to 2013 is more than 2.1 million, among which $1.6 \%$ is bus and almost $5 \%$ is truck. With the present growth rate of vehicle over $10 \%$, number of faulty vehicles is ever increasing and as a result road safety is compromised. Both physical and mechanical defects in vehicle, which no longer meet or conform to the rules and regulations of road traffic system can contribute to a crash or increase the likelihood of injuries to road users and depreciation of vehicle. According to the data collected from ARI (Accident Research Institute), from the year 1998 to 2010, among all fatal accidents $25 \%$ occurred due to bus and 3\% due to truck traffic [1]. Also in absence of periodic replacement practice of defective parts,

Corresponding author: Sakib Mahmud Khan, Ph.D. student, research fields: ITS (Intelligent Transportation System) and vehicle modification practice in developing countries. E-mail: sakibk@g.clemson.edu. number of aged vehicles is rising in road. Research conducted in Canada shows that the proportion of fatalities, serious injuries and collisions for the occupants of aged defective vehicles (15 years and older) increases and is approximately two and a half times higher than the average for occupants of all vehicles [2].

However, if vehicle physical condition is highlighted, it is critically observed from pedestrian safety analysis in Al-Ain city of the UAE (United Arab Emirates) that vehicle size and design do not affect the anatomical injury distribution and severity. In UAE, high-impact speed is dominant over vehicle type when it comes to severity and pattern of injury distribution [3]. Thus, transport systems in developed areas like UAE, London and elsewhere are actually aiming to reduce traffic speeds in order to decrease traffic mortality. Similarly, according to another research conducted in USA (United States of America), it is derived that there exist little relationship between vehicle wheelbase and casualty risk in frontal crashes [4]. However, in developing countries like Bangladesh, it is likely that vehicle 
physical defects are more often a factor in accidents as vehicle condition is generally much worse. The vehicle fleet is usually older with many vehicles imported as second-hand from other countries. And there may be difficulty in obtaining suitable spare parts. Though adequate legal provisions have been made in the "Motor Vehicle Ordinance" of Bangladesh to conform standard vehicle condition and allowable physical dimensions, unfortunately these legal provisions are neither observed properly nor enforced strictly in Bangladesh. Taking advantage of weak legal enforcement, uncontrolled vehicle modification is spreading as a contagious social disease. The unauthorized change of physical condition of the vehicle is done after buying vehicle, whether it is a fresh stock or a secondary purchased vehicle. Also, the extent of multiple periodic modification of vehicles is seriously noticeable while maintaining the vehicle or recovering it after any serious crash. This uncontrolled vehicle modification of heavy vehicles often prevents the owner from renewing fitness certificate which is considered as mandatory every year as per local constitution. Such alarming situation prevails in Bangladesh as local body makers, without any academic or institutional training, perform modification where owners mainly take the role of engineers. The scope of this paper includes the comprehensive examination of the extent of local illegal modification practice. It is important to comprehend the impact of unskilled local body workers on improper modification work. To do that appropriate share of resources is given to find out the quality of vehicle body works performed in auto workshop with a view to improve local vehicle life expectancy and performance.

\section{Why Is Vehicle Modification an Issue?}

In Europe, prevailing "Safe System Approach" acknowledges that the design of vehicles must be based on human limitations including tolerances to physical force. This is implemented through national strategic plans such as "Vision Zero" in Sweden or "Sustainable Safety" in the Netherlands. Unlike those countries, roadway safety is often compromised in Bangladesh because of excess presence of road unworthy modified vehicles. Nevertheless, "Motor Vehicles Act, 1984" presents a complete definition of vehicle modification:

"Modification in a relation to a motor vehicle means that it is no longer in conformity with the regulations when any of the items has been modified contrary to the regulations or replaced or equipped by an item which is not the prototype or not Homo-logical or when using them is considered prejudicial to safety or to the environment but shall not include wear of an item and the resulting condition" [5].

However, according to field survey unsafe modification practice of public vehicles (large buses, mini buses and human haulers) and goods vehicles (large trucks, covered trucks and medium trucks) prevails here for more than three decades. Modification practice significantly varies with vehicle types. Besides owners' profit earning motive, the situation has also worsened by the inexperienced local body makers, who do not possess any formal training or institutional skill. More importantly, as stated by the section 47 of "Motor Vehicle Ordinance 1983", every vehicle needs to renew fitness certificate every year under proper inspection of BRTA (Bangladesh Road Transport Authority). Nevertheless from survey conducted by BRTA, it was found that about $20 \%$ of the buses do not have fitness for operation [6]. Additionally, these unauthorized modified vehicles often induce roadway collision with additional body features (like sharp-edged bumpers, horizontal angles etc.). According to the collected data of ARI, among the total recorded number of vehicles causing accidents in 10 years (2000-2010) in the area under the jurisdiction of DMP (Dhaka Metropolitan Police), $17.9 \%$ vehicles were reported to have no valid fitness certificate [1]. Among the heavy vehicles, fitness 
certificate was not found for $9.8 \%$ buses, $10.7 \%$ minibuses, $13 \%$ trucks and $11.9 \%$ heavy trucks. Such gloomy findings lead to strong belief that local vehicle modification is one serious issue to investigate considering high collision rate of such vehicles.

\section{Trends and Impacts of Vehicle Modification}

Local vehicle modification is done mainly on commercial vehicles, i.e., buses or trucks to give them a distinctive appearance or to improve vehicle performance. Owners intentionally do that to add desired features in order to modify the carriage capacity without following manufacturers' instruction or any distinctive scientific approach. This incident particularly alters the vehicle center of gravity, makes them unstable and increases tyre burst accidents on road. However, Table 1 is furnished with the performed modification dimension of heavy vehicles as has been collected by field interview of body making

Table 1 Summary of field survey result of modified vehicle body configuration performed by local body-makers (for the year 2011 and 2012).

\begin{tabular}{|c|c|c|c|}
\hline Vehicle type & Vehicle classification & Model/parts & Model name/dimension \\
\hline \multirow{14}{*}{ Bus } & \multirow{5}{*}{$\begin{array}{l}\text { Large bus (more than } 40 \\
\text { seats) }\end{array}$} & Engine & Modified \\
\hline & & False chassis & Offset from chassis: $0.61 \mathrm{~m}$ (Hino), $0.91 \mathrm{~m}$ (Tata) \\
\hline & & \multirow{3}{*}{ Seat number } & Increases up to $52,57,62$ number of seats \\
\hline & & & Seat area cross section: $0.46 \mathrm{~m} \times 0.46 \mathrm{~m}$ \\
\hline & & & Seat to seat distance: $0.71 \mathrm{~m}$ \\
\hline & \multirow{9}{*}{$\begin{array}{l}\text { Mini bus (between } 16 \text { to } 39 \\
\text { seats) }\end{array}$} & Engine & Modified \\
\hline & & False chassis & Offset from chassis: $0.76-1.07 \mathrm{~m}$ \\
\hline & & & $\begin{array}{l}\text { Inner clear height: } 1.91 \mathrm{~m} \text { (if roof-top fan is not attached); } 1.83 \mathrm{~m} \text { (if } \\
\text { roof-top fan is attached) }\end{array}$ \\
\hline & & Body & Length $7.62 \mathrm{~m}$ (authorized length: $6.4 \mathrm{~m}$ ) \\
\hline & & & Width $2.19 \mathrm{~m}$ \\
\hline & & Seat number & 42 seat (authorized: 31 seat), 21 seat (authorized: 30 seat) \\
\hline & & & Width of three seats cover area: $1.14-1.22 \mathrm{~m}$ (authorized width: $1.37 \mathrm{~m}$ ) \\
\hline & & Seat dimension & Width of three seats cover area: $0.71-0.76 \mathrm{~m}$ (authorized width $0.91 \mathrm{~m}$ ) \\
\hline & & & Seat center to center distance: $0.51 \mathrm{~m}$ (authorized distance: $0.66 \mathrm{~m}$ ) \\
\hline \multirow{19}{*}{ Truck } & \multirow{9}{*}{$\begin{array}{l}\text { Heavy truck (three or more } \\
\text { axles) }\end{array}$} & False chassis & Offset from chassis: $0.76-1.22 \mathrm{~m}$ \\
\hline & & \multirow{4}{*}{ Carrier body } & Cross section of carrier: $2.44 \mathrm{~m} \times 2.44 \mathrm{~m}$ (covered truck) \\
\hline & & & Length: 5.49-6.1 m (authorized length: $5.49 \mathrm{~m}$ ) \\
\hline & & & Width: $2.44 \mathrm{~m}$ \\
\hline & & & Width extension: $0.11 \mathrm{~m}$ on both side of headroom (covered truck) \\
\hline & & \multirow{4}{*}{ External bumper } & Offset from front of headroom: $0.05 \mathrm{~m}$ \\
\hline & & & Offset from back of the carrier: $0.1 \mathrm{~m}$ \\
\hline & & & Bumper thickness: $0.005 \mathrm{~m}$ \\
\hline & & & Bumper height: $0.51-0.76 \mathrm{~m}$ \\
\hline & \multirow{7}{*}{$\begin{array}{l}\text { Medium truck (two axles } \\
\text { rigid trucks) }\end{array}$} & False chassis & Offset from chassis: $0.3-0.91 \mathrm{~m}$ \\
\hline & & \multirow{4}{*}{ Carrier body } & Cross section of carrier: $2.13 \mathrm{~m} \times 2.13 \mathrm{~m}$ (covered truck) \\
\hline & & & Length: $6.1 \mathrm{~m}$ (authorized length: $4.89 \mathrm{~m}$ ) \\
\hline & & & Width: $2.44 \mathrm{~m}$; width extension: $0.25 \mathrm{~m}$ on both side of headroom \\
\hline & & & Height of side cover of the carrier: $0.76 \mathrm{~m}$ (uncovered truck) \\
\hline & & \multirow{2}{*}{ External bumper } & Projection from front of headroom: $0.56 \mathrm{~m}$ \\
\hline & & & Bumper height: $0.76 \mathrm{~m}$ \\
\hline & \multirow{3}{*}{$\begin{array}{l}\text { Light truck (small trucks up } \\
\text { to } 3 \text { t payload) }\end{array}$} & False chassis & Projection from chassis: $0.47-0.91 \mathrm{~m}$ \\
\hline & & \multirow{2}{*}{ External bumper } & Projection from front of headroom: $0.61 \mathrm{~m}$ \\
\hline & & & Bumper height: $0.15-0.18 \mathrm{~m}$ \\
\hline
\end{tabular}


workers who are engaged in this work for more than 10 years.

From the enlisted modified dimensions of Table 1, it is clearly perceived that owners, compromising service quality, are stretching the performance of vehicle parts beyond their average life expectancies. Lack of attention towards frequent maintenance practice is one of the primary causes of modification. Moreover, more complex modification trends than past are found after the field survey of this research work. In previous research, some known practiced modifications were mentioned, like making of one type of vehicle on another type of vehicle chassis, projecting vehicle bodies beyond maximum dimension specified by the manufacturer, altering seating arrangement of bus, fixing additional features like bumpers, rooftop railing, ladder, etc. [7]. But recent research has found that many new trends of modification are invented, for example adding external metal plates in exterior and interior side of chassis of trucks, fixing additional angle on urban vehicle (specially urban buses), relocating external rear view mirrors inside of the urban vehicles, etc. [8]. Some of the observed dreadful scenarios of vehicle modification from field survey in the year 2012 are shown in Fig. 1. These risky alterations of vehicles increase extra material cost for owner, threat the vehicle's reliability, raise questions about the vehicle's legal compliance, compromise public perceptions to the service quality of the public vehicle and above all reduce the expected service life of a vehicle. All these findings of the latest survey do not come as a surprise, rather it confirms the continuity and serious deterioration in the condition of the modification practice. Considering the impacts, a grievous conclusion can be drawn without hesitation that neither modification practice has any positive effect on vehicle nor the modified vehicles can ensure traffic safety. Poor authoritative performance by BRTA can be solely considered as the responsible factor of such widespread unregulated practice. It is observed form a comprehensive research conducted on vehicle inspectors in UAE that $17 \%$ of them think private companies can help in the inspection process as the inspection center increases, which will ease on people, prevent overcrowd, and offers a better service [9]. Considering this outcome, it is high time to think about participation of private companies in heavy vehicle fitness checking procedure.

\section{Research Methodology}

Detailed field survey is conducted for one year (started from December 2011 and concluded at December 2012) in major three bus terminals in Dhaka (Gabtoli, Sayedabad and Mohakhali bus terminals) and three truck depots (Gabtoli, Tejgaon and Kamalapur truck depot) in order to reveal the degree of rampant vehicle modification. The vehicle body measurement survey is performed with measuring tape of millimeter accuracy on randomly
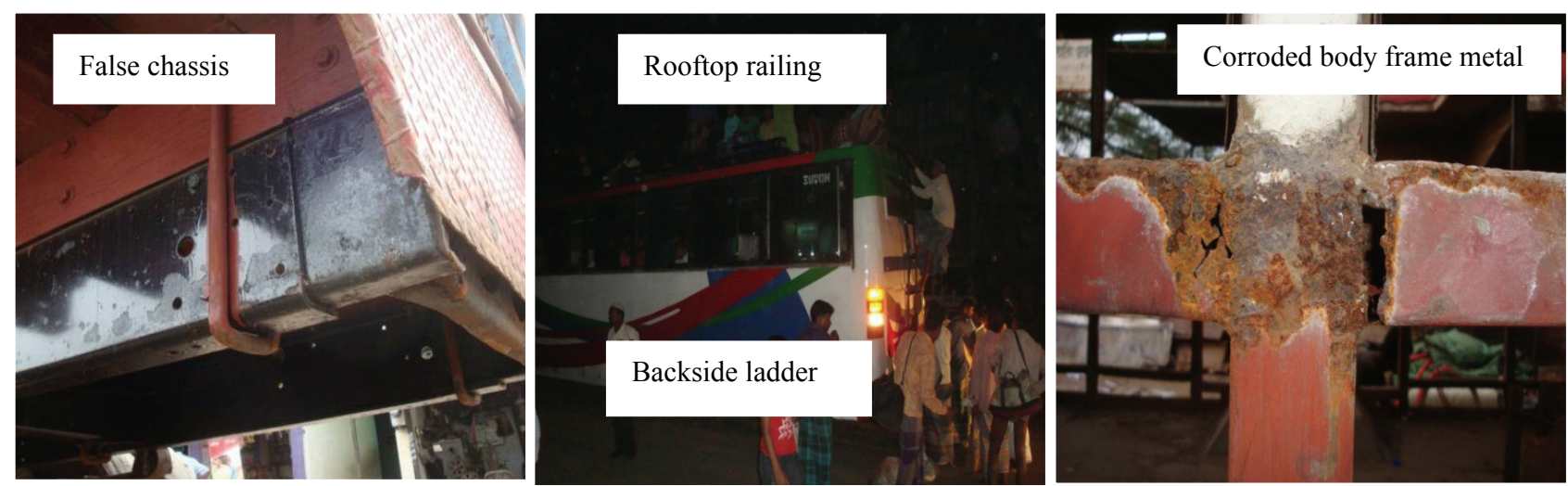

Fig. 1 Observed poor vehicle modification and maintenance practices during field survey. 
taken 30 large buses, 20 mini buses and 20 trucks (uncovered and covered trucks each). Moreover, detailed vehicle condition survey is undertaken simultaneously on same vehicle samples. Finally, 35 local body workers are interviewed based on the prepared questionnaire survey to investigate nature of the modification work.

\section{Analysis and Discussion}

\subsection{Analysis of Measured Vehicle Dimension}

Field measurement is documented keeping the allowable parameters from Motor Vehicle Rules, 1997 and Motor Vehicle Rules, 1940 in mind [10, 11]. Some statistical analysis is performed on the available vehicle dimensions from field in order to reveal most performed vehicle body configuration. Table 2 is presented here with the summary analysis of collected field data to find out the deviation of individual vehicle feature from the permitted limit. It is distinctly evident from Table 2 that as far as local vehicles are involved, violation of permitted dimension is a common practice in Bangladesh.

It is clear from Table 2 that weighted average value (7.73 ft.) of front overhang of large buses exceeds the allowable limit (6.9 ft.) by a distinct deviation of $12 \%$. Also, this table verifies the tendency of majority of vehicle owners to stretch the rear overhang by attaching false chassis on the rear side of vehicle. It is derived that the weighted average value (8.83 ft.) of rear overhangs for mini buses exceeds the allowable limit (5.92 ft.) with a maximum deviation of $49.7 \%$. Thus, almost half of the mini buses are arranged with extra space surpassing original passenger carrying capacity. Moreover, with reduced seat area (5.8\% less

Table 2 Summary of statistical analysis of local vehicle dimension (for the year 2011 and 2012).

\begin{tabular}{|c|c|c|c|}
\hline Vehicle type & Observed vehicle feature & Statistical characteristic & $\begin{array}{l}\text { Vehicle } \\
\text { measurement }\end{array}$ \\
\hline \multirow{6}{*}{$\begin{array}{l}\text { Large bus (wheel base } \\
\text { varies in } 4.72-5 \text { m range) }\end{array}$} & \multirow{3}{*}{ Front overhang (m) } & Frequency analysis range of maximum percentage (69\%) & $2.34-2.44$ \\
\hline & & Weighted average & 2.36 \\
\hline & & Allowable length & 2.1 \\
\hline & \multirow{3}{*}{ Rear overhang (m) } & Frequency analysis range of maximum percentage (50\%) & $3.34-3.64$ \\
\hline & & Weighted average & 3.79 \\
\hline & & Allowable length & 3.2 \\
\hline \multirow{6}{*}{$\begin{array}{l}\text { Mini bus (wheel base } \\
\text { varies in } 3.61-4.2 \mathrm{~m} \\
\text { range) }\end{array}$} & \multirow{3}{*}{ Seat dimension $\left(\mathrm{m}^{2}\right)$} & Frequency analysis range of maximum percentage (55\%) & $0.41-0.44$ \\
\hline & & Weighted average & 0.45 \\
\hline & & Minimum seat area & 0.48 \\
\hline & \multirow{3}{*}{ Rear overhang (m) } & Frequency analysis range of maximum percentage (60\%) & $2.54-3.01$ \\
\hline & & Weighted average & 2.69 \\
\hline & & Allowable length & 1.8 \\
\hline \multirow{7}{*}{$\begin{array}{l}\text { Covered medium truck } \\
\text { (wheel base varies in } \\
4.93-5.41 \text { m range) }\end{array}$} & \multirow{3}{*}{ Rear overhang (m) } & Frequency analysis range of maximum percentage (30\%) & $2.95-3.05$ \\
\hline & & Weighted average & 2.78 \\
\hline & & Allowable length & 2.47 \\
\hline & \multirow{2}{*}{ Bumper front projection (m) } & Frequency analysis range of maximum percentage (60\%) & $0.61-0.71$ \\
\hline & & Weighted average & 0.61 \\
\hline & \multirow{2}{*}{ Bumper height (m) } & Frequency analysis range of maximum percentage (50\%) & $0.51-0.61$ \\
\hline & & Weighted average & 0.51 \\
\hline \multirow{6}{*}{$\begin{array}{l}\text { Uncovered medium truck } \\
\text { (wheel base varies in } \\
4.19-4.8 \text { m range) }\end{array}$} & \multirow{2}{*}{$\begin{array}{l}\text { Projection of front carrier } \\
\text { beyond headroom }(\mathrm{m})\end{array}$} & Frequency analysis range of maximum percentage (45\%) & $0.2-0.305$ \\
\hline & & Weighted average & 0.22 \\
\hline & \multirow{2}{*}{ Bumper front projection (m) } & Frequency analysis range of maximum percentage (65\%) & $0.41-0.51$ \\
\hline & & Weighted average & 0.35 \\
\hline & \multirow{2}{*}{ Bumper height (m) } & Frequency analysis range of maximum percentage (50\%) & $0.61-0.71$ \\
\hline & & Weighted average & 0.61 \\
\hline
\end{tabular}


than minimum seat space), these mini buses pose an open physical threat for long route travellers, who are compelled to sit in this modified seats with limited movement for a long time interval. Another dreadful aspect of reckless modification is the external steel bumper of medium trucks. With sharp edges, these bumpers allow trucks to move on national highway as "The Killer Giant on Road".

Furthermore, Fig. 2 shows the summary of collected field data from the year 2011 to 2012. Close observation of Fig. 2 reveals the unexpected degree of poor local vehicle modification. It shows that $86.7 \%$ of the 30 large buses exceed length of front overhang while all large buses surpass the limit of rear overhang. Also, for more than three-fourth of overall sample of large buses (76.7\%) the overall width of large vehicle is found to exceed permitted dimension. However, intension of squeezing maximum number of seats is clearly perceived with limited space for legroom between seats in $60 \%$ of the 20 minibuses. For goods vehicle, $85 \%$ of the 20 covered trucks are reported to exceed allowable length of rear overhang. On the other hand, the back carrier of uncovered trucks has offset on both sides beyond truck cabin. For this research, this offset for carrier side is found to exceed limit of side overhang for $25 \%$ of the 20 uncovered trucks. All these are nothing but inevitable indicator of increasing grievous practice uncontrolled vehicle local modification practice.

\subsection{Analysis of Vehicle Condition Survey}

It is mentioned earlier that local road safety is primarily threatened by risky modification feature, like side projection of truck carrier beyond head-room and lateral angles fixed externally with truck carrier or bus. Large vehicle condition survey from local field investigation is accomplished with a view to find the extent of modification practices which rise questions regarding this safety issues and the detailed analysis is presented in Fig. 3. It is found from Fig. 3 that $20 \%$ of total surveyed 20 covered trucks and $80 \%$ of total 20 uncovered trucks are dangerously projected beyond front headroom, although any unsafe alteration of vehicle is restricted by local constitution [5]. Such projection has a direct effect on safety issue by inducing side blind spots of local trucks. According to a research conducted in United States for the period of 2003-2007, although highest number of impact for trucks occur in front side (62.5\%), side collision rate is also significant. Almost $15.5 \%$ of the total impact occurs in left hand side of the driver and $6.5 \%$ of them occur in right hand side of the driver [12]. Such impacts are more serious in developing countries like Bangladesh, as locally modified truck drivers cannot

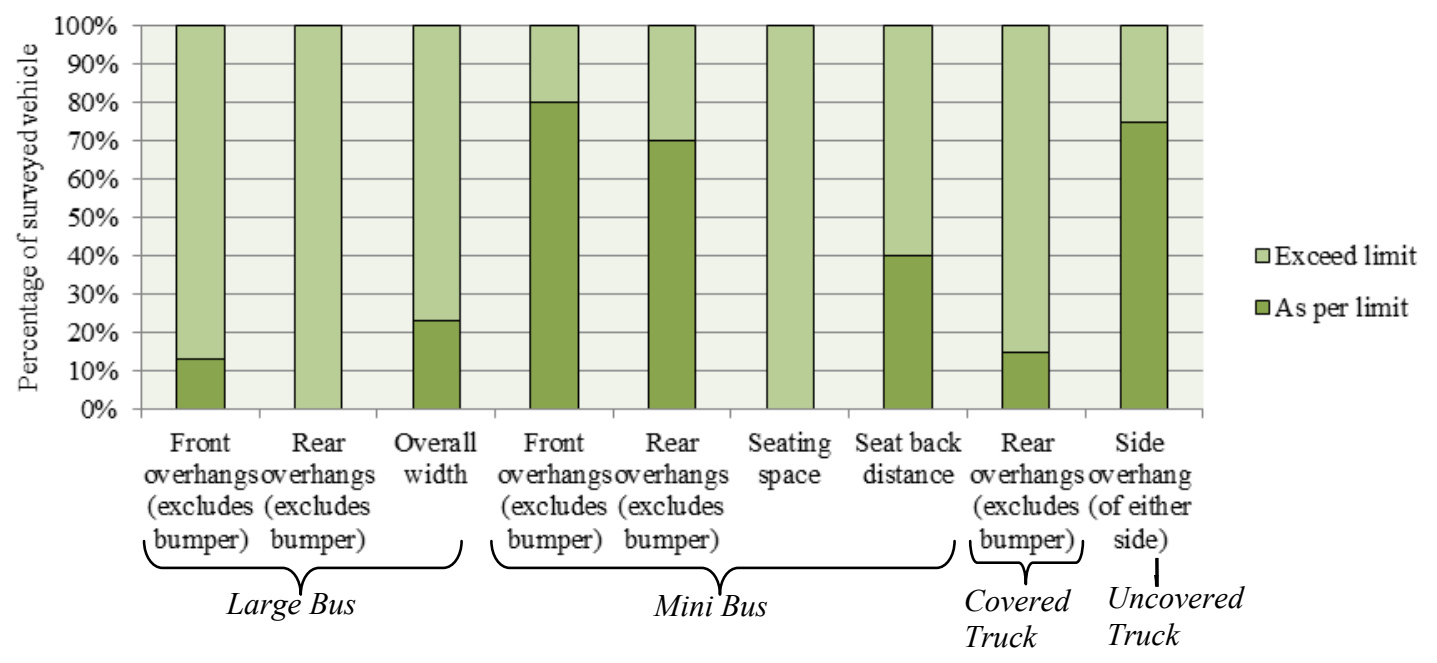

Fig. 2 Summary of measured vehicle data analysis (for the year 2011 and 2012). 


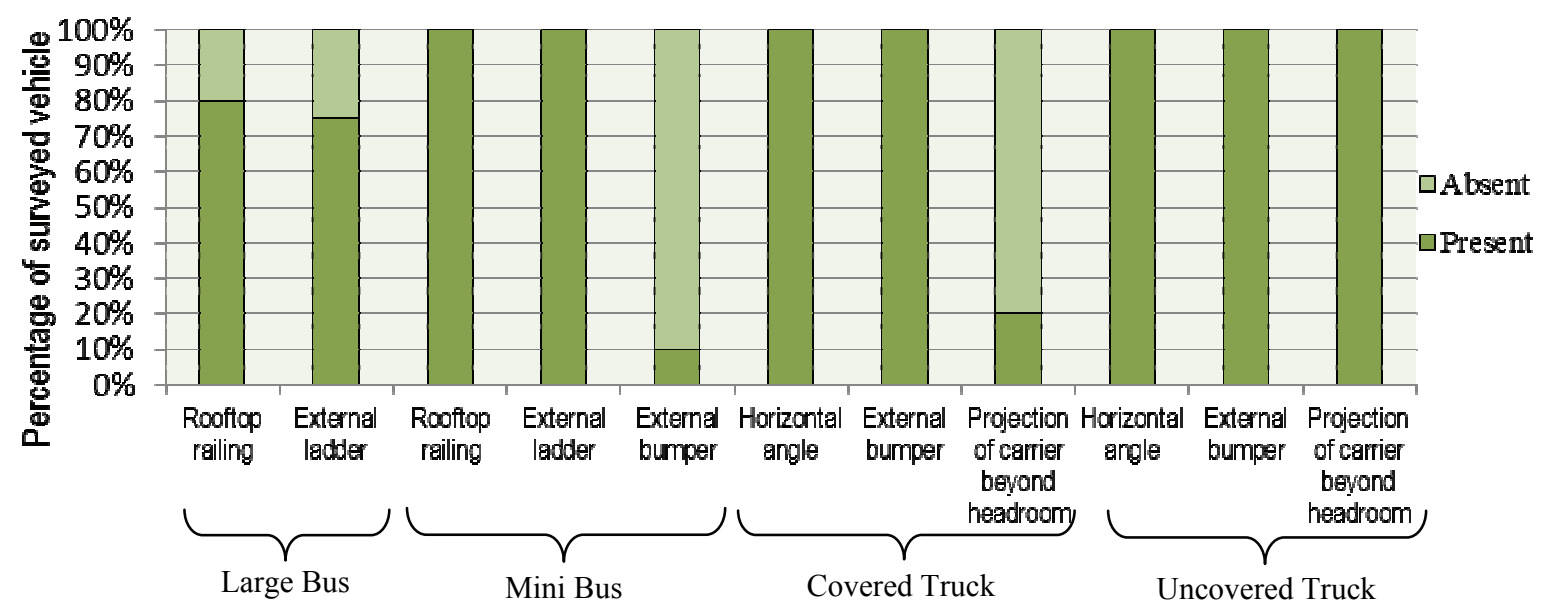

Fig. 3 Summary of vehicle condition survey result (for the year 2011 and 2012).

identify objects at an angle of $15^{\circ}$ made with the side of truck headroom or cabinet. Here Fig. 4 shows this situation for a locally operational uncovered truck which was taken during the field investigation from Gabtoli area. Besides presence of sharp edged bumper and risky steel angles in all uncovered trucks reflect the shocking extent of illegal modification. For buses, it is believed that extra features like rooftop railing encourage overloading of goods or passenger and increase potential risk of passenger falling from roof at critical locations like sharp turn or frequent undulation. According to a research on accident analysis, out of total 1,011 accidents occurred near Jamuna Multipurpose Bridge, 50 passengers' fall-down incidents happened from rooftop and most are recorded as fatal or severely injured [13]. Majority of large buses (80\%) along with all minibuses with rooftop railing in recent study disclose that, this unwanted situation of fall-down incident will continue in near future unless earnest preventive measures against illegal modification are implemented.

\subsection{Analysis of Local Vehicle Owners and Body Workers Characteristics}

Alteration of vehicle physical condition widens the scope of deviation of performance from standard vehicle. Inconsistent complex modification is the consequence of fragmented ownership of heavy vehicle fleets. Commercial vehicle owners deliberately take the risk of random alteration of vehicle parts. Field investigation exposes that scrap steel is used for altering chassis and vehicle body while
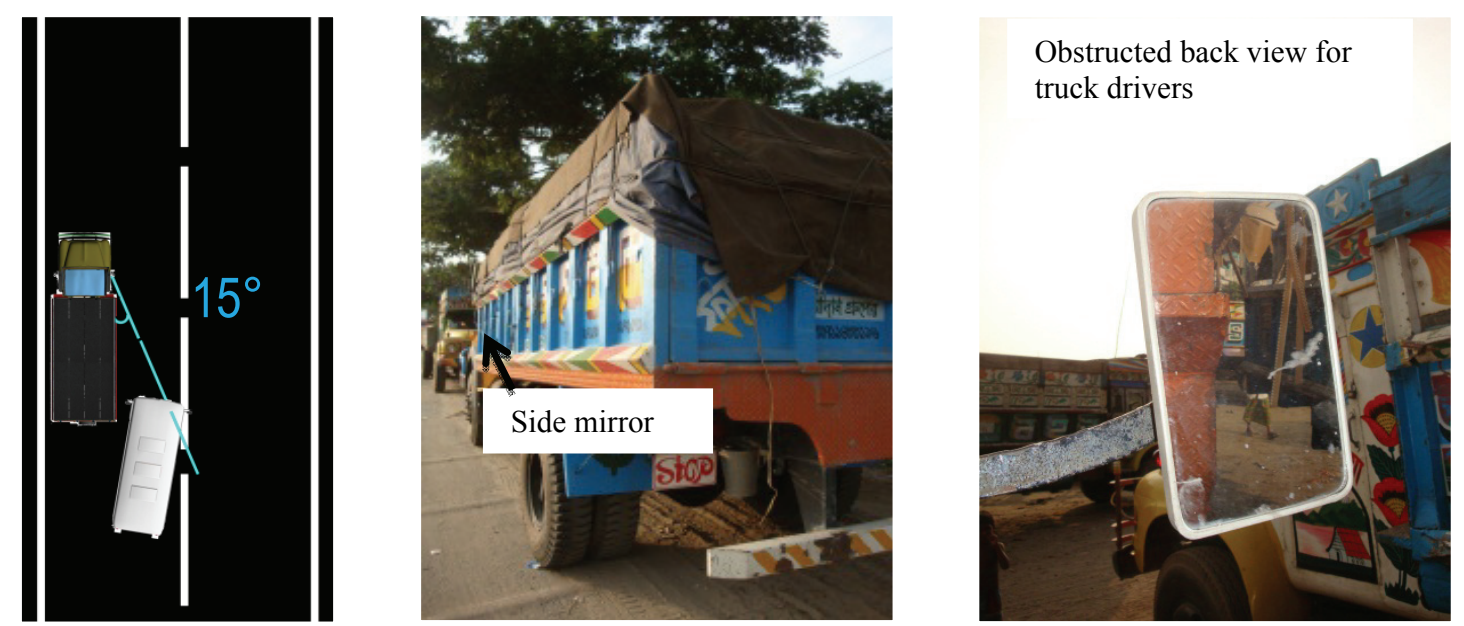

Fig. 4 Side black spot induced by projected local truck carriers. 
angular bars are mainly used for rooftop railing and in-vehicle structures like seats. Use of locally made glass instead of toughened or tempered glass has become widespread especially in window glasses of urban vehicles. As urban vehicle drivers always show aggressive attitude while moving on road, these low quality glasses pose serious threat in case of side collision. By tradition, local modification is performed by a group of naive body workers, who perform mainly under the supervision of an experienced senior. With the growing number of local workshop, number of amateur workers is increasing. During field survey, some of them are observed to perform vehicle body work alone without any guidance. Simultaneously risk is also increasing as no investigation is performed on the accomplished task, neither material checking nor inspection of welding. Hence quality of work solely depends on performance of body makers. Findings of the investigation on detailed characteristics of 35 local body workers from Dhaka are enlisted in Fig. 5 for the time range 2011 to 2012. It is distinctly visible from this figure that major portion of body workers $(80 \%)$ has no institutional skill; rather they follow the instructions from senior workers. While servicing, mainly they apply their own perception (74\%). Specification is mainly followed in renowned

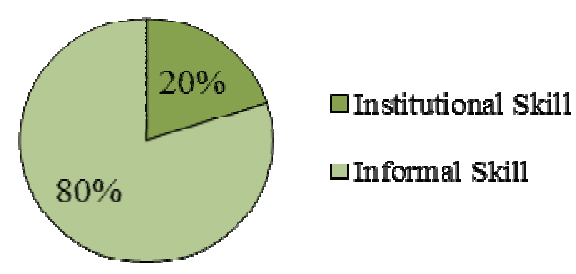

(a) Body work perform with

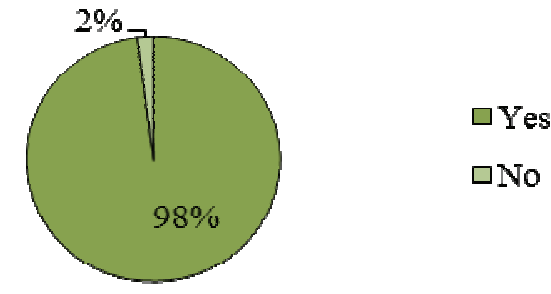

(c) Monotony of working and overtiming workshops, while in local workshop about $6 \%$ workers replicate from picture of popular models. Meanwhile being the only earning member in family, overtime working beside regular working hours $(8 \mathrm{~h})$ is preferred by all body workers. Rush of limited time $(20 \%)$ and budget restraint $(66 \%)$ accompanied by monotony of working overtime leave a wide scope of questioning the quality of works performed by body makers. Another crucial factor is the poor working environment dominating in local workshops, where workers perform in a hazardous condition without safety precautions as shown in Fig. 6 which was taken from bus workshops in Gabtoli area. No local workshops are found with adequate fast-aid kit, fire extinguishers or gas masks. Even some perform without helmet or gloves while welding work, thus serious injuries like vision problem, skin burn and noise disturbance are very common in local body workers. Another shocking aspect is the existing number of child labors performing in local vehicle body shops. It is derived from a report of UNICEF that, in the year 2002-2003 number of children (aged 5-17 years) engaged in hazardous labor (auto workshops, welding, etc.) is 1.3 million [14]. The prevailing unsafe working situation poses not only a serious threat to the normal physical growth of these

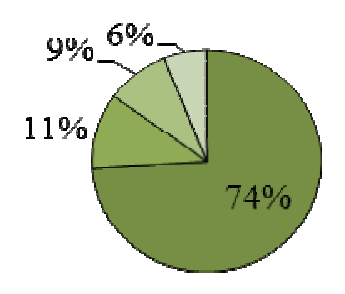

(b) Required standards

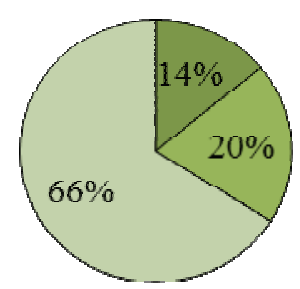

$\square$ Quality of work

$\square$ Time limit

$\square$ Budget limit

\section{$\square$ Perception \\ $\square$ Specification \\ $\square$ Blindly done \\ $\square$ Picture of renowned model}

(d) Motivation of performed work

Fig. 5 Characteristics of local vehicle body workers (for the year 2011 and 2012). 

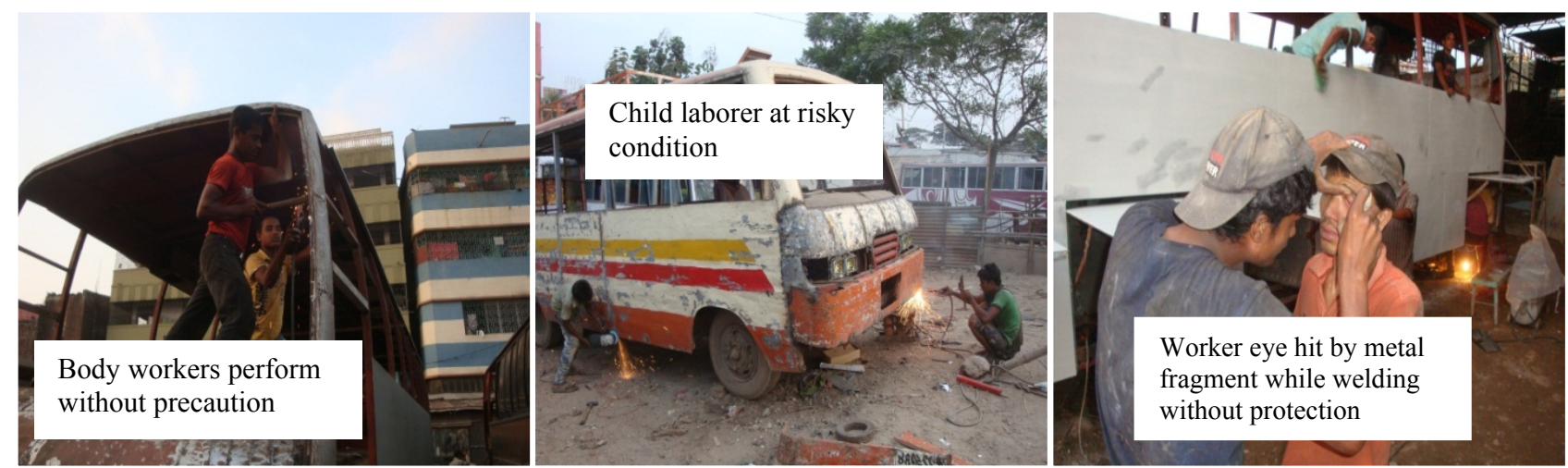

Fig. 6 Poor working environment dominating in local workshops.

children, but also many of them are found with disturbing mental disorder as an effect of working without necessary counter measures in such polluted surroundings. This in long term affects the quality of the modification work and also thus leaves a wide scope to question about the accomplished work.

\section{Conclusions and Recommendations}

As regular vehicle fitness checking is one of the main tools to control such uncontrolled vehicle modification practice, main responsibility lies with BRTA. Many of the modification features (like body angle, ladder, in-vehicle material, external bumpers etc.) are clearly identifiable. Strict vehicle fitness checking without any compromising can help to minimize the problem quickly. Recent emerging procedure of digital registration can be thought as a helping tool to regulate large vehicle fitness. Also, such grim findings from the study about degree of vehicle modification and body worker performance emphasize the need for taking instant initiatives like random roadside inspection of vehicles including the police and vehicle inspectors day and night to encourage compliance with road safety standards. Besides before further modification practices are introduced, rising safety awareness among owners and drivers about the adverse effects of reckless vehicle alternation can help for a long run. As in Bangladesh, the number of vehicle is on a rise with an alarming rate, it is becoming difficult day by day to conduct vehicle inspection with limited manpower, equipment and infrastructural setup. Often the vehicle inspection centers in Dhaka city are found under pressure of excessive presence of vehicle. Crowded centers prevent inspectors from conducting a comprehensive and effective inspection, thus many vehicle features are overlooked or ignored during local vehicle inspection. This should be avoided to assure quality control. Moreover, immediate needs of privatization and short time based fitness renewal system (like Singapore and UK) are suggested by local law enforcing authority for a better service quality. Uniformity of testing equipment like exhaust gas analyzers, measurement of headlights focus, side slip testers, brake testers, and tire quality device is needed to be prioritized to have arrest the rising number of aged road-unworthy vehicles. To do that, law for breaking down the tradition of fragmented ownership of vehicle fleet is to be established. Similarly, strict law enforcement is to be confirmed to encourage the owners to consolidate the vehicle fleet. Most importantly adequate academic knowledge and institutional training should be made obligatory for local body makers. Age verification of workers by government and workshop owners is needed to be prioritized to stop the rising number of child laborers. As the extent of modification is continuing for a good time span, the regulatory body and law enforcing authorities are to be blamed equally. Legal provisions for standard vehicle feature and vehicle maintenance practice have been made in the Motor Vehicle Ordinance, but unfortunately they are not enforced 
strictly in Bangladesh. No doubt exists about the upcoming challenges in near future for concerned authorities; however strong determination and co-operation of both government authority and vehicle owners will help to overcome the current situation in near future.

\section{Acknowledgments}

The authors express their gratitude to $\mathrm{Mr}$. Md. Barek Ullah Khan for funding the research work.

\section{References}

[1] ARI Database, 1998-2010 Accident Data Collected from Different Police Ranges and Metropolitan Offices, Accident Research Institute, BUET, Dhaka, 2012.

[2] J.F. Lécuyer, A. Chouinard, Study on the effect of vehicle age and the importation of vehicles 15 years and older on the number of fatalities, serious injuries and collisions in Canada, in: Proceedings of the Canadian Multidisciplinary Road Safety Conference XVI, Manitoba, Canada, 2006.

[3] E.M. AlEassa, H.O. Eid, F.M.A. Zidan, Effects of vehicle size on pedestrian injury pattern and severity: Prospective study, World Journal of Surgery 37 (1) (2013) 136-140.

[4] T. Wenzel, Analysis of the Relationship between Vehicle Weight/Size and Safety, and Implications for Federal Fuel Economy Regulation, Final report prepared for the Office of Energy Efficiency and Renewable Energy, US Department of Energy, Berkeley, CA, 2010.

[5] Motor Vehicles Rules, Government of the People's
Republic of Bangladesh, Ministry of Communication, 1984.

[6] S. Hasnine, Evaluation and development of bus based public transport in Dhaka city, in: Proceedings of 4th Annual Paper Meeting and 1st Civil Engineering Congress, Dhaka, Bangladesh, 2011, pp. 217-226.

[7] M.S. Hoque, The vehicle component: Vehicle factor and vehicle safety, in: Road Safety Training Course on Understanding Road Accident Problems and Their Remedies, Accident Research Center, BUET, Dhaka, 2003.

[8] S.M. Khan, M.S. Hoque, Analysis of extent and impact of vehicle modification, in: Proceedings of the $1 \mathrm{st}$ International Conference on Advances in Civil Engineering, Chittagong, Bangladesh, 2012.

[9] M.Y.E. Selim, M.A. Maraqa, Y.E. Hawas, A.M.O. Mohamed, Assessing vehicular inspection centers using multiple decision-making criteria, in: Proceedings of the World Congress on Engineering, London, UK, 2011.

[10] Motor Vehicle Rules, Government of the People's Republic of Bangladesh, Ministry of Communication, 1940.

[11] Motor Vehicle Rules, Government of the People's Republic of Bangladesh, Ministry of Communication, 1997.

[12] N. Bezwada, S. Dissanayake, Characteristics of fatal truck crashes in the United States, in: Proceedings of the 2009 Mid-continent Transportation Research Symposium, Ames, Iowa, 2009.

[13] M.S. Hoque, M.R. Hasan, Involvement of vehicle factors in road accidents, Journal of Civil Engineering 35 (1) (2007) 17-27.

[14] UNICEF, Child Labour in Bangladesh, 2010, http://www.unicef.org/bangladesh/Child_labour.pdf (accessed Nov. 1, 2013). 